\title{
REFERENCES
}

1. H.-J. Hoehnke, Zur Theorie der Gruppoide. I, Math. Nachr. 24 (1962), 137168; III, Acta Math. 13 (1962), 91-100.

2. A. H. Clifford and G. B. Preston, The algebraic theory of semigroups, Math. Surveys No. 7, Amer. Math. Soc., Providence, R. I. 1961.

TUlane UNIVERSITY

\section{EVERY STANDARD CONSTRUCTION IS INDUCED BY A PAIR OF ADJOINT FUNCTORS}

H. KLEISLI

In this note, we prove the converse of the following result of P. Huber [2]. Let $F: \mathfrak{K} \rightarrow \mathscr{L}$ and $G: \mathfrak{L} \rightarrow \mathcal{K}$ be covariant adjoint functors, that is, functors such that there exist two (functor) morphisms $\zeta: I \rightarrow G F$ and $\eta: F G \rightarrow I$ satisfying the relations

$$
\begin{aligned}
& (\eta * F) \circ(F * \zeta)=\imath * F \\
& (G * \eta) \circ(\zeta * G)=\imath * G .
\end{aligned}
$$

Then, the triple $(C, k, p)$ given by

$$
C=F G, \quad k=\eta \quad \text { and } \quad p=F * \zeta * G
$$

is a standard construction in $\mathcal{L}$, that is, $C$ is a covariant functor, $k: C \rightarrow I$ and $p: C \rightarrow C^{2}$ are (functor) morphisms, and the following relations hold:

$$
\begin{aligned}
& (k * C) \circ p=(C * k) \circ p=\imath * C, \\
& (p * C) \circ p=(C * p) \circ p .
\end{aligned}
$$

This standard construction is said to be induced by the pair of adjoint functors $F$ and $G$. For further explanation of the notation and terminology, see [2], or the appendix of [1].

TheOREM. Let $(C, k, p)$ be a standard construction in a category $\mathfrak{L}$. Then there exists a category $\Re$ and two covariant functors $F: \mathfrak{K} \rightarrow \mathfrak{L}$ and $G: \mathfrak{L} \rightarrow \mathfrak{K}$ such that

(i) $F$ is (left) adjoint to $G$,

(ii) $(C, k, p)$ is induced by $F$ and $G$.

Received by the editors March 2, 1964. 
The category $\mathscr{K}$ is given as follows. The objects of $\mathscr{K}$ are the same as those of $\mathcal{L}$. For each pair $A, A^{\prime}$ of objects, we define

$$
\operatorname{Hom} \varkappa\left(A, A^{\prime}\right)=\operatorname{Hom} \&\left(C A, A^{\prime}\right) \text {. }
$$

For each triple $A, A^{\prime}, A^{\prime \prime}$ of objects, and each pair of morphisms $\alpha \in \operatorname{Hom}_{\mathcal{K}}\left(A, A^{\prime}\right)$ and $\alpha^{\prime} \in \operatorname{Hom}_{\mathcal{K}}\left(A^{\prime}, A^{\prime \prime}\right)$, the composition, $\alpha^{\prime} \cdot \alpha \in \operatorname{Hom}_{\mathfrak{K}}\left(A, A^{\prime \prime}\right)$ is given by

$$
\alpha^{\prime} \cdot \alpha=\alpha^{\prime} \circ C \alpha \circ p A \text {. }
$$

The identity ${ }_{A} \in \operatorname{Hom}_{\mathcal{K}}(A, A)$ is defined by setting

$$
\mathrm{l}_{A}=k A: C A \rightarrow A \text {. }
$$

The associativity and identity laws follow from (4) and (3). By (4), we have

$$
\begin{aligned}
\alpha^{\prime \prime} \cdot\left(\alpha^{\prime} \cdot \alpha\right) & =\alpha^{\prime \prime} \circ C\left(\alpha^{\prime} \circ C \alpha \circ p A\right) \circ p A \\
& =\alpha^{\prime \prime} \circ C \alpha^{\prime} \circ C^{2} \alpha \circ((C * p) \circ p) A \\
& =\alpha^{\prime \prime} \circ C \alpha^{\prime} \circ C^{2} \alpha \circ((p * C) \circ p) A \\
& =\left(\alpha^{\prime \prime} \circ C \alpha^{\prime} \circ p A^{\prime}\right) \circ C \alpha \circ p A=\left(\alpha^{\prime \prime} \cdot \alpha^{\prime}\right) \cdot \alpha .
\end{aligned}
$$

By (3), $\alpha \cdot \iota_{A}=\alpha \circ C k A \circ p A=\alpha \circ((C * k) \circ p) A=\alpha \circ(\iota * C) A=\alpha$, and, similarly, $\iota_{A} \cdot \alpha=\alpha$.

The functor $C$ can be factored as follows:

$$
\begin{aligned}
& \mathscr{L} \stackrel{C}{\longrightarrow} \mathcal{L}, \\
& G \searrow{ }_{\mathscr{K}} \nearrow F
\end{aligned}
$$

where $G$ and $F$ are covariant functors given by $G A=A$ and $G \alpha=\alpha \circ k A$ for every object $A$ and morphism $\alpha$ of $\mathcal{L}, F B=C B$ and $F \beta=C \beta \circ p B$ for every object $B$ and morphism $\beta$ of $\mathcal{H}$. The functor properties of $G$ and $F$ are immediate consequences of (3), (4) and of the definition of the identities in $\mathscr{K}$. The verifications are straightforward.

In order to show that $F$ is (left) adjoint to $G$, and that $(C, k, p)$ is induced by $F$ and $G$, put $\eta=k$ and define $\zeta B=\iota_{C B}: C B \rightarrow C B$ for every object $B$ of $\Re$. The family $(\zeta B)_{B \in \mathcal{K}}$ yields a (functor) morphism $\zeta: I \rightarrow G F$. Indeed, let $\beta \in \operatorname{Hom}_{\mathcal{K}}\left(B, B^{\prime}\right)$; then, $\zeta B^{\prime} \cdot \beta$ $=\iota_{C B^{\prime}} \circ C \beta \circ p B=C \beta \circ p B=C \beta \circ C_{C B} \circ p B=G F \beta \cdot \zeta B$. Clearly, $C=F G$, and by definition $k=\eta$. Moreover, for each object $A$ of $\mathcal{L}$,

$$
(F * \zeta * G) A=F_{\iota C G A}=C_{\iota C A} \circ p A=p A ;
$$


hence $p=F * \zeta * G$. Using (3), we obtain

$$
\begin{aligned}
((\eta * F) \circ(F * \zeta)) * G & =(\eta * F G) \circ(F * \zeta * G)=(k * C) \circ p \\
& =\iota * C=(\iota * F) * G .
\end{aligned}
$$

It is easily seen that the factor $G$ may be cancelled. Thus, relation (1) holds. Furthermore, we have

$$
\begin{aligned}
F *((G * \eta) \cdot(\zeta * G)) & =(F G * \eta) \circ(F * \zeta * G)=(C * k) \circ p \\
& =\iota * C=F *(\iota * G) .
\end{aligned}
$$

Here, the factor $F$ may be cancelled. Indeed, let $\beta_{1}$ and $\beta_{2}$ be elements of $\operatorname{Hom}_{\mathcal{K}}\left(B, B^{\prime}\right)$ such that $F \beta_{1}=F \beta_{2}$. By (3),

$$
\begin{aligned}
k B^{\prime} \circ F \beta_{1} & =k B^{\prime} \circ C \beta_{1} \circ p B=\beta_{1} \circ k C B \circ p B=\beta_{1} \circ((k * C) \circ p) B \\
& =\beta_{1} \circ(\iota * C) B=\beta_{1},
\end{aligned}
$$

and, similarly, $k B^{\prime} \circ F \beta_{2}=\beta_{2}$. Hence $\beta_{1}=\beta_{2}$. Therefore, relation (2) holds.

\section{REFERENCES}

1. R. Godement, Theorie des faisceaux, Actualités Sci. Indust. No. 1252, Hermann, Paris, 1958. 385.

2. P. Huber, Homotopy theory in general categories, Math. Ann. 144 (1961), 361-

UNIVERSITY OF OTTAWA 